\title{
NGHIÊN CỬU CHI TIẾT ĐỘ CAO CỦA MẠT GEOID CỤC BỘ HÒN DẤU SO VỚI MẠT GEOID TOÀN CẦU TRÊN LÃNH THỔ VIÊTT NAM
}

\author{
ThS. NGUYẼ̃N TUẤN ANH
}

Viện Khoa học Đo đạc và Bản đồ

\section{Tóm tắt:}

Bài báo đề cập đến việc đánh giá chi tiết độ cao của mặt Geoid cục bộ Hòn Dấu sát nhất với mặt biển trung bình nhiều năm tại trạm nghiệm triểu Hòn Dấu so với mặt Geoid toàn cầu dựa trên các điểm trọng lực chi tiết trên lãnh thổ Việt Nam. Các kết quả tính toán cho thấy độ cao của mặt Geoid cục bộ Hòn Dấu so với mặt Geoid toàn cầu bằng $0.890 \mathrm{~m}$ và không đổi trên toàn bộ lãnh thổ Việt Nam.

\section{1. Đặt vấn đề}

Với mục đích nghiên cứu xây dựng mô hình Quasigeoid quốc gia khởi đầu VIGAC2014 (National Preliminary Quasigeoid VIGAC2014) đối với lãnh thổ Việt Nam dựa trên mô hình trọng trường Trái đất EGM2008, chúng ta phải giải quyết bài toán chuyển mô hình dị thường độ cao toàn cầu EGM2008 tương ứng với mặt Quasigeoid toàn cầu về mặt Quasigeoid cục bộ Hòn Dấu. Để giải quyết bài toán nêu trên, trong tài liệu (Hà Minh Hòa (2007)) đã xây dựng được phương trình quan hê tương đương giữa độ cao chuẩn toàn cầu $\bar{H}_{M}^{\gamma}$ (độ cao của điểm $\mathrm{M}$ trên mặt vật lý Trái đất so với mặt Quasigeoid toàn cầu) và độ cao chuẩn cục bộ Hòn Dấu $\bar{H}_{M}^{\gamma}$ ở dạng sau:

$$
\bar{H}_{M}^{\gamma}=H_{M}^{\gamma}+\frac{\bar{W}_{0}-W_{0}}{\overline{\bar{\gamma}}_{M}},
$$

và phương trình quan hệ tượng đương giữa dị thường độ cao toàn cầu $\bar{\zeta}_{M}$ của điểm (độ cao của điểm $Q$ nằm trên mặt Quasigeoid toàn cầu tương ứng với điểm $\mathrm{M}$ so với mặt ellipsoid WG.S84 quốc tế) và dị thường độ cao cục bộ $\zeta_{M}$ (độ cao của điểm $\mathrm{P}$ nằm trên mặt Quasigeoid cục bộ tương ứng với điểm M so với mặt Ellipsoid WGS84 quốc tế) ở dạng sau:

$$
\zeta_{M}=\bar{\zeta}_{M}+\frac{\bar{W}_{0}-W_{0}}{\overline{\bar{\gamma}}_{M}},
$$

Ngày nhận bài: 15/8/2015 ở đây $\quad \bar{W}_{0}=62636856,0 \mathrm{~m}^{2} \cdot \mathrm{s}^{-} \quad$ là thế trọng trường của mặt Geoid toàn cầu (xem Petit G. and Luzum B. (2010)) được sử dụng để xây dựng mô hình EGM2008 (Tenzer $R$., Vatrt V. and Amos M. (2009)); $W_{0}$ - thế trọng trường của mặt Geoid cục bộ sát nhất với mặt biển trung bình nhiều năm tại trạm nghiệm triều Hòn Dấu; $\overline{\bar{\gamma}}_{M}$ - giá trị trung bình của gia tốc lực trọng trường chuẩn (đơn vị $\mathrm{m} . \mathrm{s}^{-2}$ ) tại điểm $\mathrm{M}$ và được xác định theo công thức:

$$
\overline{\bar{\gamma}}_{M}=\hat{\gamma}_{0}-10^{-5} \cdot\left(0,1543 \cdot H_{M}^{\gamma}+0,036 \cdot 10^{-6} \cdot\left(H_{M}^{\gamma}\right)^{2}\right) \text {, }
$$

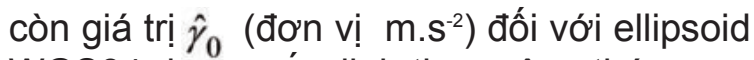
WGS84 được xác định theo công thức:

$$
\begin{array}{r}
\hat{\gamma}_{0}=9,7803253359 \cdot\left(1+0,00530248 \cdot \sin ^{2} B-\right. \\
\left.0,0000058497 \cdot \sin ^{2} 2 B\right)<m \cdot s^{-2}>
\end{array}
$$

Trong công thức (1) và (2) đại lượng

$$
\Delta \zeta_{M}=\frac{\bar{W}_{0}-W_{0}}{\overline{\bar{\gamma}}_{M}}
$$

là độ cao của dıem $r$ tren mặt Quasigeoid cục bộ tương ứng với điểm $\mathrm{M}$ so với mặt Quasigeoid toàn cầu (xem Hình 1). Khi ký hiệu $H_{M}$ là độ cao trắc địa của điểm $\mathrm{M}$ và được xác định từ kết quả đo đạc và xử lý các kêt quả đo đạc GNSS trong ITRF tương ứng với Ellipsoid quốc tế WGS-84, lưu ý $H_{M}=\bar{H}_{M}^{\gamma}+\bar{\zeta}_{M}=H_{M}^{\gamma}+\zeta_{M}$, từ quan hệ ở công thức (1) chúng ta sẽ suy ra quan hệ (2).

Ngày chấp nhận đăng: 31/8/2015 


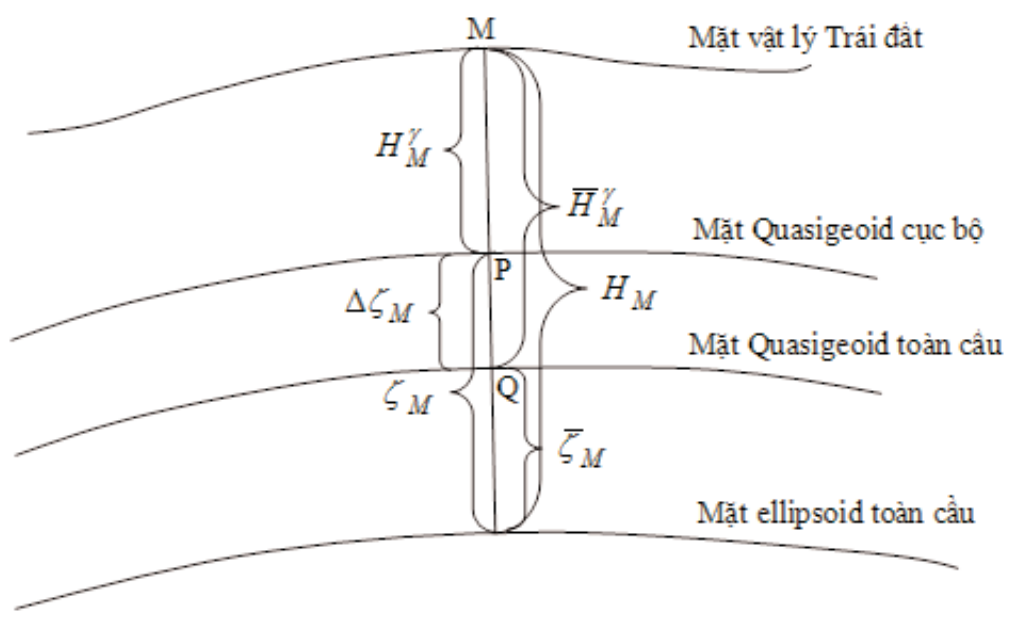

Hình 1: Quan hệ giữa các mặt Quasigeoid, mặt vật lý Trái đất và mặt Ellipsoid

Một số kết quả tính toán thực nghiệm đã này bằng

$$
\Delta \zeta_{M}=\frac{8,7089 \mathrm{~m}^{2} \cdot \mathrm{s}^{-2}}{\overline{\bar{\gamma}}_{M}}=0,890 \mathrm{~m}
$$

được công bố trong các tài liệu (Hà Minh

Hòa (2012); Hà Minh Hòa (2013); Hà Minh Hòa (2014)). Dựa trên 35 điểm độ cao hạng I quốc gia được phân bố tương đối đều trên lãnh thổ Việt Nam, thêm vào đó trên các điểm độ cao hạng I nêu trên có đo GPS và các dữ liệu đo GPS được xử lý trong ITRF2008, các dị thường độ cao $\bar{\zeta}$ của các điểm độ cao này được xác định từ mô hình EGM2008, đã xác định được thế trọng trường $W_{0}=62636847,2911 \mathrm{~m}^{2} \cdot \mathrm{s}^{-2}$ của mặt Geoid cục bộ Hòn Dấu với sai số trung phương $\pm 0,183 \mathrm{~m}^{2} \mathrm{~s}^{-2}$. Đồng thời, đại lượng $\Delta \zeta_{M}=\frac{\bar{W}_{0}-W_{0}}{\overline{\bar{\gamma}}_{M}}$ được đánh giá trên 40 điểm trọng lực quốc gia (11 điểm trọng lực cơ sở, 29 điểm trọng lực hạng I) phủ trùm lãnh thổ Việt Nam được phân bố từ vùng núi cao, trung $\mathrm{du}$, đồng bằng và các đảo (Bạch Long Vĩ, Phú Quốc). Kết quả cho thấy đại lượng và không đổi trên toàn bộ lãnh thổ Việt Nam.

Để kiểm tra kết luận (4) ở mức chi tiết trên lãnh thổ Việt Nam, trong bài báo này sẽ thực hiện tính toán theo công thức (4) trên 133 điểm trọng lực chi tiết được phân bố trong 09 vùng phủ trùm lãnh thổ Việt Nam.

\section{Giải quyết vấn đề}

Ưu điểm cơ bản của việc xác định độ cao của mặt Geoid cục bộ Hòn Dấu so với mặt Geoid toàn cầu dựa trên công thức (4) nằm ở chỗ các giá trị $\Delta \zeta_{M}$ được tính toán không bị ảnh hưởng của sự xê dịch của điểm $\mathrm{M}$ trên mặt vật lý Trái đất do các tác nhân tự nhiên (chuyển dịch đứng của vỏ Trái đất, sạt lở đất v.v...) và các tác nhân nhân sinh (xê dịch mốc do mở rộng các đường giao thông, xây dựng các khu công nghiệp và đô thị v.v...). Thật vậy, giá trị $\overline{\bar{\gamma}}_{M}$ trong công thức (3) thay đổi nhỏ bỏ qua dưới sự thay đổi của độ cao chuẩn $H_{M}^{\gamma}$. Giả sử với độ xê dịch $\Delta H_{M}^{\gamma}$ của điểm $M$ là $10 \mathrm{~m}$, tức độ cao chuẩn của điểm $\mathrm{M}$ bị thay đổi đi $10 \mathrm{~m}$ do các tác nhân tự nhiên và nhân sinh (điều này không bao giờ xẩy ra trong thực tế), thành phần thứ hai ở vế phải chỉ ảnh hưởng đến số hạng thứ năm sau dấu phẩy của giá trị 
$\overline{\bar{\gamma}}_{M}$, còn thành phần thứ ba ở vế phải chỉ ảnh hưởng đến số hạng thứ 12 sau dấu phẩy của giá trị $\overline{\bar{\gamma}}_{M}$. Do đó việc đánh giá độ cao Geoid cục bộ Hòn Dấu so với mặt Geoid toàn cầu theo công thức (4) cho kết quả tin cậy nhất.

Dữ liệu đưa vào tính toán giá trị trung bình của gia tốc lực trọng trường chuẩn $\overline{\bar{\gamma}}$ và kiểm tra độ chênh giữa Quasigeoid cục bộ và Quasigeoid toàn cầu $\Delta \zeta$ là các điểm trọng lực chi tiết tại 9 khu vực đặc trưng phân bố đều khắp lãnh thổ Việt Nam, các điểm trọng lực chi tiết này được lấy ra từ CSDL trường trọng lực Việt Nam và được chọn ở các vị trí địa hình khác nhau trong mỗi khu vực. Kết quả thu được như bảng sau: (xem bảng)

Theo biểu thức (3), (4) thì giá trị $\Delta \zeta$ gần như không phụ thuộc nhiều vào vị trí và độ cao điểm xét, điều này đồng nghĩa với việc độ cao của điểm bất kỳ trên Quasigeoid cục bố Hòn Dấu so với Quasigeoid toàn cầu là hẳng số trên toàn lãnh thố Việt Nam. Kết quả tính toán kiểm tra tại các điểm trọng lực

\begin{tabular}{|c|c|c|c|c|c|}
\hline STT & Tên điểm & Vĩ độ ( $\left.{ }^{\circ}\right)$ & $H^{y}(\mathrm{~m})$ & y $\left(\mathrm{m} \cdot \mathrm{s}^{-2}\right)$ & $n \leq(\mathrm{m})$ \\
\hline$I$ & \multicolumn{5}{|c|}{ Tây Bắc } \\
\hline 1 & B71 & 21.745195 & 373 & 978684.0695 & 0.8899 \\
\hline 2 & TH-HT12 & 21.745356 & 1543 & 978503.6292 & 0.8900 \\
\hline 3 & A158 & 21.745983 & 621 & 978645.8609 & 0.8899 \\
\hline 4 & A99 & 21.746233 & 771 & 978622.7389 & 0.8899 \\
\hline 5 & TH-HT5 & 21.748472 & 499 & 978664.8351 & 0.8899 \\
\hline 6 & A237 & 21.749405 & 1051 & 978579.7503 & 0.8900 \\
\hline 7 & A128 & 21.755462 & 633 & 978644.5985 & 0.8899 \\
\hline 8 & A156 & 21.755713 & 566 & 978654.9493 & 0.8899 \\
\hline 9 & TH-HT10 & 21.761133 & 736 & 978629.0630 & 0.8899 \\
\hline 10 & 1763 & 21.763199 & 89 & 978729.0043 & 0.8898 \\
\hline 11 & BL-TL5 & 21.767280 & 1117 & 978570.6821 & 0.8900 \\
\hline 12 & A94 & 21.768563 & 1002 & 978588.4976 & 0.8899 \\
\hline 13 & TH-HT11 & 21.769545 & 744 & 978628.3518 & 0.8899 \\
\hline 14 & A91 & 21.769633 & 560 & 978656.7398 & 0.8899 \\
\hline 15 & B72 & 21.769920 & 734 & 978629.9175 & 0.8899 \\
\hline 16 & A150 & 21.770871 & 1240 & 978551.9368 & 0.8900 \\
\hline II & \multicolumn{5}{|c|}{ Đông Bắc } \\
\hline 1 & $\mathrm{R} 740$ & 22.588356 & 1157 & 978616.3033 & 0.8899 \\
\hline 2 & B61 & 22.588751 & 391 & 978734.4797 & 0.8898 \\
\hline 3 & D174 & 22.590436 & 457 & 978724.4057 & 0.8898 \\
\hline 4 & D253 & 22.593139 & 266 & 978754.0451 & 0.8898 \\
\hline 5 & B82 & 22.593748 & 261 & 978754.8555 & 0.8898 \\
\hline 6 & B82 & 22.598509 & 225 & 978760.7144 & 0.8898 \\
\hline 7 & D236 & 22.602068 & 306 & 978748.4455 & 0.8898 \\
\hline 8 & $\mathrm{D} 68$ & 22.604235 & 1008 & 978640.2989 & 0.8898 \\
\hline 9 & TL16 & 22.609980 & 203 & 978764.8432 & 0.8899 \\
\hline 10 & B97 & 22.611737 & 202 & 978765.1100 & 0.8898 \\
\hline 11 & B63 & 22.612731 & 470 & 978723.8278 & 0.8898 \\
\hline 12 & R443 & 22.619346 & 345 & 978743.5354 & 0.8898 \\
\hline 13 & $\mathrm{D} 13$ & 22.619811 & 300 & 978750.5076 & 0.8898 \\
\hline 14 & D243 & 22.626821 & 440 & 978729.3585 & 0.8898 \\
\hline 15 & R738 & 22.628723 & 1052 & 978635.0817 & 0.8898 \\
\hline
\end{tabular}


Nghiên cứu

\begin{tabular}{|c|c|c|c|c|c|}
\hline IIII & \multicolumn{5}{|c|}{ Tây Tây Bắc } \\
\hline 1 & $\mathrm{~B} 80 \mathrm{a}$ & 21.365301 & 902 & 978579.0461 & 0.8900 \\
\hline 2 & B51 & 21.367668 & 1143 & 978542.0225 & 0.8900 \\
\hline 3 & A102 & 21.367990 & 476 & 978644.9214 & 0.8899 \\
\hline 4 & D96 & 21.368120 & 979 & 978567.3428 & 0.8900 \\
\hline 5 & D98 & 21.370237 & 777 & 978598.6283 & 0.8900 \\
\hline 6 & A119 & 21.375484 & 961 & 978570.5699 & 0.8899 \\
\hline 7 & A113 & 21.376465 & 510 & 978640.1954 & 0.8899 \\
\hline 8 & A127 & 21.377420 & 496 & 978642.4136 & 0.8900 \\
\hline 9 & A131 & 21.379107 & 534 & 978636.6549 & 0.8899 \\
\hline 10 & D38 & 21.382636 & 977 & 978568.5403 & 0.8900 \\
\hline 11 & B35 & 21.384038 & 437 & 978651.9207 & 0.8899 \\
\hline 12 & $\bar{D} 50$ & 21.387101 & 1220 & 978531.3381 & 0.8899 \\
\hline 13 & B64 & 21.387730 & 1348 & 978511.6381 & 0.8900 \\
\hline$\overline{\text { IV }}$ & \multicolumn{5}{|c|}{ Đông Đông Bắc } \\
\hline 1 & h160 & 20.758712 & 126 & 978662.0498 & 0.8899 \\
\hline 2 & 1287 & 20.759199 & 6 & 978680.5943 & 0.8899 \\
\hline 3 & g75 & 20.759362 & 94 & 978667.0260 & 0.8899 \\
\hline 4 & H168 & 20.761080 & 99 & 978666.3572 & 0.8899 \\
\hline 5 & H164 & 20.761275 & 275 & 978639.2145 & 0.8899 \\
\hline 6 & h162 & 20.762588 & 146 & 978659.1957 & 0.8899 \\
\hline 7 & g73 & 20.767253 & 92 & 978667.8065 & 0.8899 \\
\hline 8 & Al299 & 20.767962 & 9 & 978680.6555 & 0.8899 \\
\hline 9 & h156 & 20.771183 & 102 & 978666.4987 & 0.8899 \\
\hline 10 & H175 & 20.772568 & 331 & 978631.2504 & 0.8899 \\
\hline 11 & g58 & 20.772910 & 90 & 978668.4535 & 0.8899 \\
\hline 12 & g55 & 20.773379 & 101 & 978666.7843 & 0.8899 \\
\hline 13 & H171 & 20.773971 & 356 & 978627.4775 & 0.8899 \\
\hline 14 & h148 & 20.775440 & 83 & 978669.6849 & 0.8899 \\
\hline $\mathbf{V}$ & \multicolumn{5}{|c|}{ Bắc Trung Bộ } \\
\hline 1 & M226 & 18.389615 & 20 & 978543.5299 & 0.8900 \\
\hline 2 & M161 & 18.392055 & 49 & 978539.1871 & 0.8900 \\
\hline 3 & M183 & 18.393402 & 77 & 978534.9395 & 0.8900 \\
\hline 4 & P42 & 18.394633 & 688 & 978440.7456 & 0.8901 \\
\hline 5 & K85 & 18.395650 & 30 & 978542.3129 & 0.8900 \\
\hline 6 & P45 & 18.396706 & 211 & 978514.4432 & 0.8900 \\
\hline 7 & M158 & 18.404152 & 164 & 978522.0970 & 0.8900 \\
\hline 8 & K48 & 18.404301 & 5 & 978546.6377 & 0.8900 \\
\hline 9 & M219 & 18.406139 & 15 & 978545.1941 & 0.8900 \\
\hline 10 & K108 & 18.410330 & 31 & 978542.9518 & 0.8900 \\
\hline 11 & M157 & 18.412462 & 85 & 978534.7351 & 0.8900 \\
\hline 12 & P36 & 18.414402 & 402 & 978485.9324 & 0.8900 \\
\hline 13 & P38 & 18.414923 & 235 & 978511.7249 & 0.8900 \\
\hline 14 & K75 & 18.419935 & 16 & 978545.7856 & 0.8900 \\
\hline 15 & M101 & 18.423371 & 69 & 978537.7937 & 0.8900 \\
\hline VI & \multicolumn{5}{|c|}{ Trung Bộ } \\
\hline 1 & R34 & 16.530471 & 26 & 978446.6438 & 0.8901 \\
\hline 2 & B360 & 16.537678 & 245 & 978413.2088 & 0.8901 \\
\hline 3 & Z196 & 16.537798 & 5 & 978450.2446 & 0.8901 \\
\hline 4 & RZ70 & 16.543243 & 7 & 978450.2040 & 0.8901 \\
\hline 5 & $\mathrm{Z12}$ & 16.548191 & 1 & 978451.3734 & 0.8901 \\
\hline 6 & B222 & 16.549849 & 381 & 978392.8262 & 0.8901 \\
\hline 7 & B224 & 16.550177 & 455 & 978381.4264 & 0.8901 \\
\hline 8 & RZ72 & 16.550261 & 8 & 978450.3952 & 0.8901 \\
\hline 9 & B340 & 16.562837 & 143 & 978430.1850 & 0.8901 \\
\hline 10 & B165 & 16.565829 & 384 & 978393.1507 & 0.8901 \\
\hline
\end{tabular}


Nghiên cứu

\begin{tabular}{|c|c|c|c|c|c|}
\hline 11 & R34A & 16.567495 & 11 & 978450.7814 & 0.8901 \\
\hline 12 & B258 & 16.575410 & 255 & 978408.8964 & 0.8901 \\
\hline 13 & R48 & 16.578401 & 109 & 978436.1982 & 0.8901 \\
\hline 14 & B203 & 16.578443 & 89 & 978439.2861 & 0.8901 \\
\hline VII & \multicolumn{5}{|c|}{ Nam Trung Bộ } \\
\hline 1 & Q62 & 14.290995 & 37 & 978341.5107 & 0.8902 \\
\hline 2 & P26 & 14.291739 & 640 & 978248.5146 & 0.8903 \\
\hline 3 & Q25 & 14.292493 & 319 & 978298.0663 & 0.8902 \\
\hline 4 & U145 & 14.292635 & 1581 & 978103.4321 & 0.8904 \\
\hline 5 & U154 & 14.293553 & 553 & 978262.0132 & 0.8902 \\
\hline 6 & N41 & 14.293938 & 5 & 978346.5752 & 0.8902 \\
\hline 7 & N11 & 14.294699 & 40 & 978341.2076 & 0.8902 \\
\hline 8 & Q35 & 14.294807 & 80 & 978335.0404 & 0.8902 \\
\hline 9 & P72 & 14.295259 & 740 & 978233.2414 & 0.8903 \\
\hline 10 & R55 & 14.295799 & 499 & 978270.4402 & 0.8902 \\
\hline 11 & R49 & 14.299043 & 255 & 978308.2228 & 0.8902 \\
\hline 12 & U29 & 14.300443 & 975 & 978197.2191 & 0.8902 \\
\hline 13 & P84 & 14.302234 & 183 & 978319.4690 & 0.8902 \\
\hline 14 & N31 & 14.303024 & 27 & 978343.5727 & 0.8903 \\
\hline 15 & TL52 & 14.307383 & 7 & 978346.8469 & 0.8902 \\
\hline VIII & \multicolumn{5}{|c|}{ Tây Nguyên } \\
\hline 1 & K12 & 12.065207 & 821 & 978131.5015 & 0.8904 \\
\hline 2 & LZ26 & 12.071686 & 21 & 978255.1561 & 0.8902 \\
\hline 3 & Z223 & 12.081695 & 1 & 978258.6113 & 0.8902 \\
\hline 4 & HJ24 & 12.088442 & 731 & 978146.2405 & 0.8903 \\
\hline 5 & JAIII34 & 12.095460 & 396 & 978198.1766 & 0.8903 \\
\hline 6 & LZ29 & 12.116106 & 10 & 978258.4941 & 0.8903 \\
\hline 7 & L33 & 12.137463 & 56 & 978252.1872 & 0.8902 \\
\hline 8 & KL158 & 12.177697 & 486 & 978187.3401 & 0.8903 \\
\hline 9 & LZ36 J & 12.206129 & 4 & 978262.7623 & 0.8902 \\
\hline 10 & LZ33 & 12.208294 & 15 & 978261.1457 & 0.8903 \\
\hline 11 & KL155 & 12.227142 & 515 & 978184.7081 & 0.8902 \\
\hline 12 & MZ11 & 12.233918 & 26 & 978260.4042 & 0.8902 \\
\hline 13 & KH68 & 12.244098 & 891 & 978127.3435 & 0.8902 \\
\hline 14 & LZ36 & 12.252741 & 7 & 978264.0392 & 0.8903 \\
\hline 15 & L38 & 12.276279 & 44 & 978259.2111 & 0.8902 \\
\hline 16 & KL147 & 12.313006 & 586 & 978176.9705 & 0.8904 \\
\hline 17 & KJ74 & 12.327407 & 902 & 978128.7699 & 0.8902 \\
\hline $\mathrm{IX}$ & \multicolumn{5}{|c|}{ Nam Bộ } \\
\hline 1 & A62 & 10.277106 & 3 & 978196.4324 & 0.8903 \\
\hline 2 & AF11 & 10.280176 & 1 & 978196.8382 & 0.8903 \\
\hline 3 & $A C 2 B$ & 10.282769 & 4 & 978196.4574 & 0.8903 \\
\hline 4 & E279 & 10.284069 & 0 & 978197.1158 & 0.8903 \\
\hline 5 & GH6B & 10.964694 & 79 & 978207.1605 & 0.8903 \\
\hline 6 & BE22 & 10.968912 & 14 & 978217.3318 & 0.8903 \\
\hline 7 & G05 & 10.969934 & 133 & 978199.0051 & 0.8903 \\
\hline 8 & G64 & 10.970452 & 124 & 978200.4112 & 0.8903 \\
\hline 9 & F48 & 10.971573 & 21 & 978216.3413 & 0.8903 \\
\hline 10 & G043 & 10.973897 & 21 & 978216.4196 & 0.8903 \\
\hline 11 & F89 & 10.974371 & 6 & 978218.7501 & 0.8903 \\
\hline 12 & E9 & 10.979251 & 5 & 978219.0688 & 0.8903 \\
\hline 13 & HE121 & 10.983021 & 30 & 978215.3384 & 0.8903 \\
\hline 14 & E10 & 10.983922 & 8 & 978218.7634 & 0.8903 \\
\hline
\end{tabular}


chi tiết có độ cao khác nhau phân bố trên toàn quốc đã xác định được hăng số này là $0,890 \mathrm{~m}$. Điều này rất thuận lợi cho việc giải quyết hàng loạt bài toán khoa học - kỹ thuật như xác định mô hình Quasigeoid quốc gia khởi đầu VIGAC2014 từ mô hình EGM2008 và chuyển đổi mô hình Địa hình mặt biển động lực trung bình DTU10 MDT trên vùng biển Việt Nam về hệ độ cao quốc gia (xem chi tiết các tài liệu Hà Minh Hòa (2012); Hà Minh Hòa (2013); Hà Minh Hòa (2014)).

\section{Kết luận}

Qua việc nghiên cứu cơ sở lý thuyết, tham khảo các tài liệu (Hà Minh Hòa (2007), Hà Minh Hòa (2012), Hà Minh Hòa (2013), Hà Minh Hòa (2014)) và thực nghiệm tính toán độ cao của các điểm trên Quasigeoid cục bộ Hòn Dấu so với mặt Quasigeoid toàn cầu tại 133 điểm trọng lực chi tiết được phân bố trong 9 vùng đặc trưng địa hình khác nhau ở Việt Nam chúng ta thấy rằng các kết quả tính toán một lần nữa khẳng định độ cao của các điểm trên Quasigeoid cục bộ Hòn Dấu so với mặt Quasigeoid toàn cầu trên toàn bộ lãnh thổ Việt Nam luôn chênh nhau 1 đại lượng không đổi là 0,890 $\mathrm{m}$ tại bất kỳ vị trí nào. $\mathrm{O}$

\section{Tài liệu tham khảo}

[1]. Hà Minh Hòa (2007). Giải quyết một số vấn đề liên quan đến việc chuyển hệ độ cao được xác định từ mặt nước biển trung bình ở trạm thủy triều về mặt Quasigeoid toàn cầu. Tạp chí Địa chính số 2 , tháng 4/2007, trg. 3 - 11.

[2]. Hà Minh Hòa (2012). Nghiên cứu xác định thế năng trọng trường thực $\mathrm{W}_{0}$ của mặt Geoid cục bộ trùng với mặt biển trung bình tại trạm nghiệm triều Hòn Dấu. Báo cáo khoa học. Tuyển tập báo cáo Hội nghị Khoa học và Công nghệ "Trắc địa và Bản đồ vì sự nghiệp tài nguyên và Môi trường". Viện Khoa học Đo đạc và Bản đồ - Hội Trắc địa, Bản đồ và Viễn thám Việt Nam. Hà Nội Tháng 10/2012. Trg. 6-19.

[3]. Ha Minh Hoa (2013). Estimating the geopotential value $\mathrm{W}_{0}$ of the local Geoid based on data from local and global normal heights of GPS/Leveling points in Vietnam. Geodesy and Cartography. Taylor \& Francis. UDK 528.21, doi: 10.3846/20296991.2013.823705, V.39 (3): 99-105.

[4]. Hà Minh Hoà (2014). Lý thuyết và thực tiễn của Trọng lực trắc địa. NXB Khoa học và Kỹ thuật, 592 trg., Hà Nội - 2014.

[5]. Petit G., Luzum B. (Eds) (2010). IERS Conventions (2010). IERS Technical Note No. 36, Verlag des Bundesamts für Kartographie und Geodäsie. Frankfurt am Main 2010, 179 pp.

[6]. Tenzer R., Vatrt V. and Amos M. (2009). Realization of the World Height System in New Zealand: Preliminary Study, Proceedings of the 2009 IAG Symposium: Geodesy for Planet Earth, Buenos Aires, Argentina, 31 August - 4 September 2009. International Association of Geodesy Symposia, Vol. 136, 2012, S. C. Kenyon, M. C. Pacino, U. J. Marti (eds), Springer, pp 343-349.0

\section{Summary}

\section{Detailed research of the Hon Dau local Geoid height relative to the global Geoid on the territory of Vietnam}

The article refers to the detailed assessment of the Hon Dau Geoid elevation closest to the mean sea level for many years on Hon Dau tide gauge station relative to the global Geoid based on detailed gravity points on territory of Vietnam. The calculation results show that the elevation of the Hon Dau local Geoid is equal to $0.890 \mathrm{~m}$ and unchanged on the whole territory of Vietnam. O 